\section{Sintese das hemoglobinas A2, S e C nos três primeiros meses após o nascimento}

Maria L.P. Nascimento

Coordenadora do Serviço de Assistência às Anemias Genéticas do Hospital Geral Roberto Santos (HGRS) Salvador, Babia

Consultora Científica dos Programas de Anemias da Universidade do Estado da Babia (UNEB)

\section{Sr. Editor,}

Do arquivo do Laboratório de Rastreamento Neonatal da APAE/Salvador, em trabalho anterior (1), 70 casos com heterozigose para as Hemoglobinas S (AS $=27$ casos $)$ e $\mathrm{C}(\mathrm{AC}=43$ casos $)$, foram separados por idade em três grupos: 1 mês $(1 \mathrm{M})=53$ casos, 2 meses $(2 \mathrm{M})=12$ casos e 3 meses $(3 \mathrm{M})=5$ casos. Através de coleta sangüínea (EDTA) havia sido feita a identificação e quantificação das hemoglobinas por método automatizado HPLC (Programas Sickle Cell e BetaThalassemia do Sistema Bio-Rad Variant Analisador de Hemoglobinas), encontrando-se os seguintes resultados para a presença das Hemoglobinas A2, S, C, A e F:

Freqüência de casos com ausência de Hb A2

\begin{tabular}{l|c|c}
\hline 1 mês & 2 meses & 3 meses \\
$20.8 \%$, & $0.0 \%$ & $0.0 \%$ \\
\hline
\end{tabular}

Valores médios para as diversas bemoglobinas

\begin{tabular}{l|l|l|l}
\hline & 1 mês & 2 meses & 3 meses \\
Hb A2\% & $1.0( \pm 0.5)$ & $1.6( \pm 0.5)$ & $3.2( \pm 0.5)$ \\
$H b$ S\% & $13.7( \pm 6.1)$ & $20.7( \pm 1.8)$ & $35.6( \pm 5.2)$ \\
\hline Hb C\% & $14.0( \pm 6.2)$ & $23.6( \pm 7.3)$ & $32.9( \pm 3.6)$ \\
Hb A \% & $15.3( \pm 9.1)$ & $25.6( \pm 8.2)$ & $41.6( \pm 6.9)$ \\
\hline$b F \%$ & $70.2( \pm 14.7)$ & $50.2( \pm 13.8)$ & $21.2( \pm 9.7)$ \\
\hline
\end{tabular}

Entre os 3 grupos (1M x 2M, 1M x 3M e 2M x 3M), as diferenças das médias foram Significantes (Kruskal Wallis $\mathrm{H}, \mathrm{p}<0.05)$ para as $\mathrm{Hb} \mathrm{A} 2, \mathrm{Hb} \mathrm{S}, \mathrm{Hb} \mathrm{C}, \mathrm{Hb} \mathrm{A}$ e Hb F.

\section{HemoglobinaA2}

Em relação à presença da $\mathrm{Hb}$ A2 antes e após o nascimento, alguns autores referem que: $\mathrm{Hb}$ A2 começa a ser sintetizada a partir da $25^{\underline{a}}$ semana da gestação em concentrações reduzidas, no sangue do cordão umbilical encontramos valores de 0.0 a $1.8 \%$ e após o nascimento, esta produção aumenta, se estabiliza no sexto mês de vida, alcançando o nível adulto com um ano de idade (2, 3, 4).

Para Hb A2 em adulto, são estes os valores referidos para métodos com maior sensibilidade na quantificação dos diferentes tipos de hemoglobinas, HPLC $(5,6)$ e Cromatografia de troca aniônica (7): Papadea et al $(5)=2.2$ a $3.0 \%$, Fucharoen et al $(6)=$ $2.6 \%( \pm 0.4)$ e Rocha et al $(7)=3.0( \pm 0.9)$.

A partir dos resultados no início deste texto (1), observamos que: somente encontramos ausência de $\mathrm{Hb}$ A2 até $1 \mathrm{M}$ em $20.8 \%$ dos casos $(\mathrm{N}=11)$; a partir de $2 \mathrm{M}$ todos os casos tiveram presença de $\mathrm{Hb} \mathrm{A} 2$, sendo que com 3M já encontramos valores médios semelhantes aos referidos para os adultos $(5,6,7)$.

\section{Hemoglobinas AS e Hb AC}

Bauer, et al (3) referem que os adultos AS tem em média 20 a $40 \%$ de Hb S. Segundo Naoum, P.C. (2) os AS tem valores entre 25 a $45 \%$. Os resultados no início deste texto (1) mostram que aos três meses de idade (3M) já encontramos valores médios de $\mathrm{Hb} \mathrm{S}$ e $\mathrm{Hb} \mathrm{C}$ que estão situados dentro das faixas referidas para os adultos $(2,3)$.

A partir dos resultados para $\mathrm{HB} \mathrm{A} 2, \mathrm{Hb} \mathrm{S}$ e $\mathrm{HB} \mathrm{C}$, no início deste texto (1), e do confronto com os valores para os adultos $(4,5,6,7)$, é provável que a partir dos três meses de idade, já podemos encontrar os valores de $\mathrm{Hb}$ A2, $\mathrm{Hb} \mathrm{S}$ e $\mathrm{Hb} \mathrm{C}$ dentro dos limites normais para adultos e não somente após os seis meses e ou um ano de idade, como referem alguns autores $(2,3,4)$.

\section{A2, S, and C hemoglobin synthesis in the three first months of life \\ Maria L.P. Nascimento}

\section{Abstract}

A prospective study of A2, S and C Hemoglobins was made in 70 beterozygous infant cases. Our results suggest, after a comparison of published studies of adults, that the normal adult levels of $\mathrm{Hb} \mathrm{A} 2, \mathrm{Hb} S$ and $\mathrm{Hb} C$ have been 
reached by the age of three months and not 6 months to one year as previously thought.

\section{Referências Bibliográficas}

1. Nascimento M.L.P, Santos H.M.G.P., Fontes M.I.M.M., Purificação A.C., Prates S. Lactentes AS e AC: Sintese das hemoglobinas A2, Se C nos três primeiros meses de idade. XXIV Congresso Brasileiro de Hematologia e Hemoterapia, pg. 94 - Rio de Janeiro, 22 a 26 de maio, 2000.

2. Naoum P.C. Diagnóstico das Hemoglobinopatias. Sarvier Editora de Livros Médicos Ltda., S. Paulo, 1987.

3. Bauer J.D., Ackerman P.G., Toro G. Clinical laboratory methods. Eighth Edition. The C. V. Mosby Company, Saint Louis, 1974.

4. Weatherall D.J. Clinica Hematológica: Hemoglobinas Anormales. Volume 2. Número 2. Salvat Editores, S. A., Barcelona (España), 1976.

5. Papadea C., Cate J.J. Identification and quantification of hemoglobins $A, F, S$ and $C$ by automated chromatography. Clin. Chem. 42:1, 5763, 1996.

6. Fucharoen S., Winichagoon P., Wisedpanichkij R., Sae-Ngow B., Sriphanich R., Oncoung W.,
Muangsapaya W., Chowthaworn J., Kanokokpongsakdi S., Bunyaratvej A., Piankijagum A., Dewaele C. Prenatal and postnatal diagnoses of thalassemias and hemoglobinopathies by HPLC. Clin. Chem. 44:4, 740-748, 1998.

7. Rocha H.H.G., Santos C.E.J., Gama A.C. Estudo comparativo de duas metodologias para determinação da Hemoglobina A2. Rev. Bras. Hematol. Hemoter. 21 (2): 89-90, 1999.

Recebido: 25/05/01

Aceito: 26/07/01

Correspondência: Maria de Lourdes Pires Nascimento Hospital Geral Roberto Santos - Serviço de Assistência as Anemias Genéticas (SAAG)

Estrada do Saboeiro, $s / n^{-}$- Cabula

CEP: 41180.780. Salvador. BA

E-mail: mlpnascimento @ e-net.com.br. 